\title{
A versatile apparatus for measuring the frequencies and durations of animal and human responses
}

JOSEPH B. SIDOWSKI, 1 UNIVERSITY OF SOUTH FLORIDA, Tampa, Florida 33620, and CARL SPEARS, SAN DIEGO STATE COLLEGE, San Diego, Califormia 92115

A versatile solid-state instrument is described that allows for the measurement of response frequencies and durations from animal or human $S$ s.

The sensitive relay apparatus described here is a highly sensitive solid-state device with numerous applications for counting and timing animal and human responses. The instrument has four channels which allow the $E$ a number of choices in recording. These include the ability to measure frequency and duration of a particular response made by four separated Ss, the reactions made by a single $\mathrm{S}$ to four different stimuli, e.g., a circle, square, triangle, and elipse, or counting and timing the number of responses "sensed" by four measurement devices. The latter might include a photocell, a moisture sensor, a standard switch, and a time-delay device.

The apparatus is depicted in Figs. 1, 2, and 3 , representing the input (front), throughput (schematic), and output (single channel only), respectively. Operation of the instrument is simplicity itself. With reference to $\mathrm{Fig.}$, the equipment is plugged into any $115-\mathrm{V}$ ac, $60 \cdot \mathrm{Hz}$ outlet and then turned on with the ac power switch located on the right side of the front panel. Inputs are connected to any or all of the four input channels (A and $B$ receptacles) and the sensitivity control

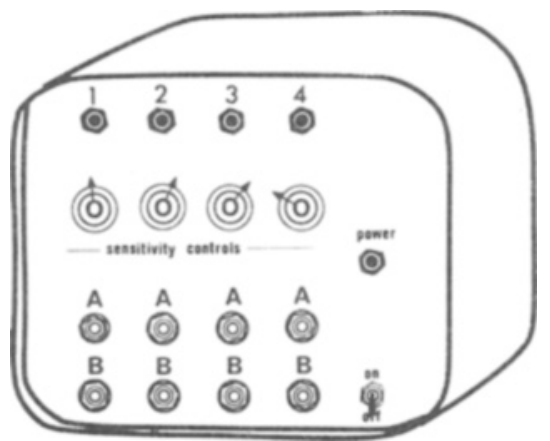

Fig. 1. Front view of chassis showing individual inputs ( $A$ and $B$ ) to the four channels with sensitivity controls and power switch. adjusted until the relay closes, at which time the indicator light glows brightly and the equipment is ready to record. Three basic types of input devices may be used: a voltage source, a resistor, and/or a switch.

For purposes of description, this discussion will focus on one channel. The three remaining channels (see Fig. 2) operate in the same manner and merely expand capabilities.

The versatility of the basic circuit is illustrated in Fig. 4. The monkey pictured in Drawing 1 is touching a metal toy to complete the circuit. Each time contact is made, the responses are recorded automatically on a digital counter and the duration accumulated on a timer wired to a terminal strip at the output side of the box, as shown in Fig. 3. The toy is merely attached to a conductor, the other end of which is plugged into Input A of the apparatus outside the cage (Fig. 1). An alligator clip at the end of another wire from Input $B$ is attached to the cage out of the animal's sight and reach. The $S$, of course, must be in contact with the cage to complete the connection. The touchplate diagrammed in Drawing 2 operates in the same manner, i.e., the relay closes when the $S$ contacts the stimulus and the response is recorded at the output. A touchplate switch is illustrated, but a moisture sensor can be used as a touchplate also.

Illustration 3 represents a sensing circuit that can be activated with a drop or two of moisture. Circuit 4 is more sensitive and operates with only a trace; normal hand or paw moisture is generally sufficient. Diagram 5 can be used with an external switch. The resistor is unnecessary but can be utilized as a safety device to limit base current on the input transistor. The resistance sensor in 6 is for high resistance values; 7 is for low values. Circuit 8 senses de voltages.

Time-delay circuits are shown in Schematics 9,10 , and 11 . Two of these, 9

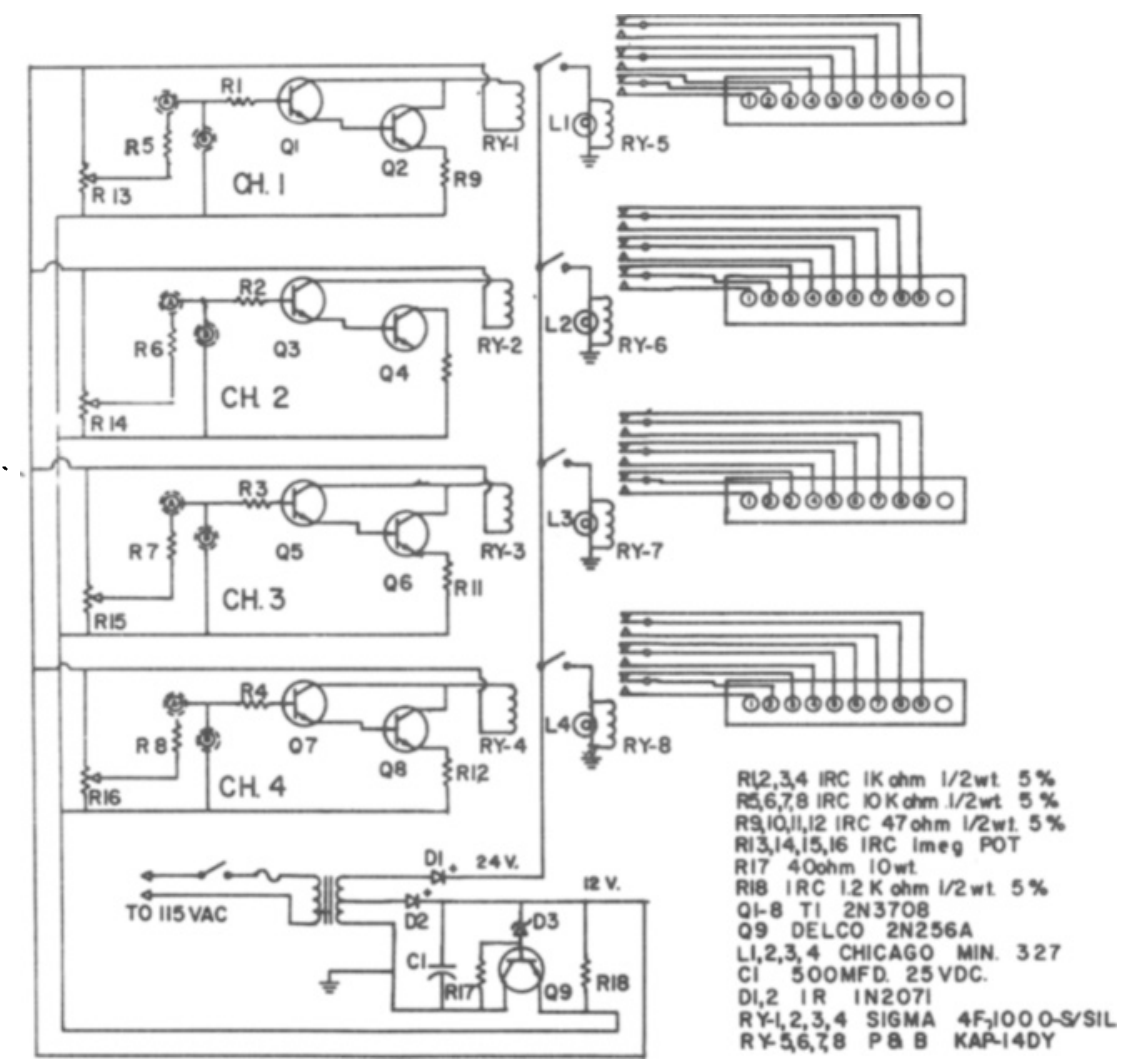

Fig. 2. Schematic of sensing relay circuit showing four channels. ${ }^{3}$ 


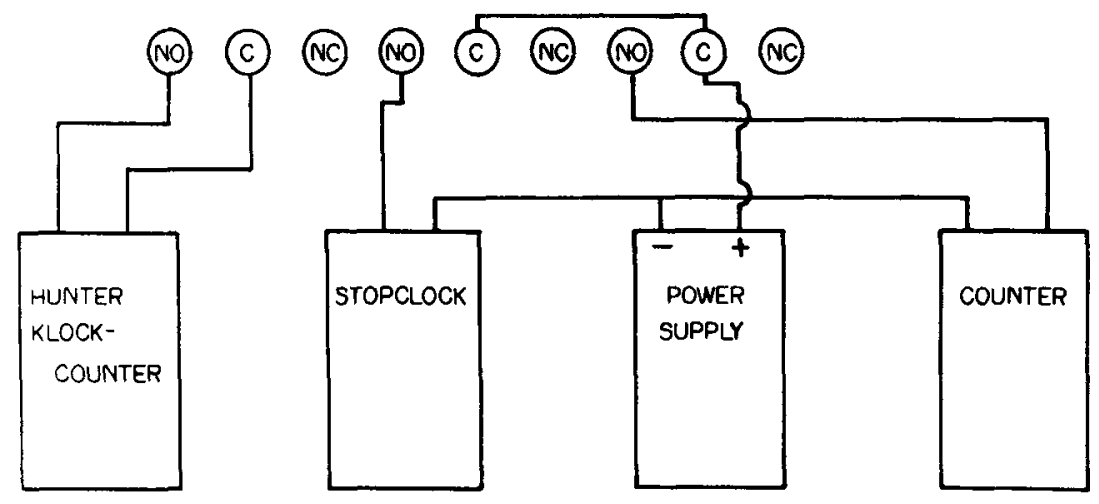

Fig. 3. Output terminal strip for a single channel showing connections for three simple response measuring devices: a Hunter KlockKounter, a Standard Electric clock with dry cell battery power supply, and a digital counter.

and 10 , delay with a momentary switch. When the switch is closed and released, the relay holds for $\mathrm{RC}$ time and releases at the end of the interval. Circuit 11 is off until the switch is activated, following which the relay remains in the off state for $\mathrm{RC}$ time.
At the end of this time interval, the relay energizes.

Diagrams 12,13,14, and 15 illustrate ways in which different photocells may be used to operate the relay.
THE CIRCUIT

The sensitive apparatus diagrammed in Fig. 2 is essentially a two-transistor, high-gain, direct-coupled dc amplifier driving a standard sensitive electromagnetic relay. In Channel 1, Transistors Q1 and Q2 (Texas Instruments planar amplifier transistors at $\$ .45$ each) are connected in a modified Darlington circuit. Basically, the Darlington circuit has two or more transistors connected in such a manner as to have a single input, a common load, and a current gain that is the product of the current gain of each transistor.

The series resistor, R1, limits base current to prevent accidental damage to the transistors by excessively strong input signals. Unbypassed emitter resistor, R9, stabilizes circuit operation; it also provides a degree of temperature compensation. Sensitivity control, R13, and current-limiting resistor, $\mathrm{R} 5$, are part of a bias and control circuit to permit the use of external resistive- and switch-type sensors.

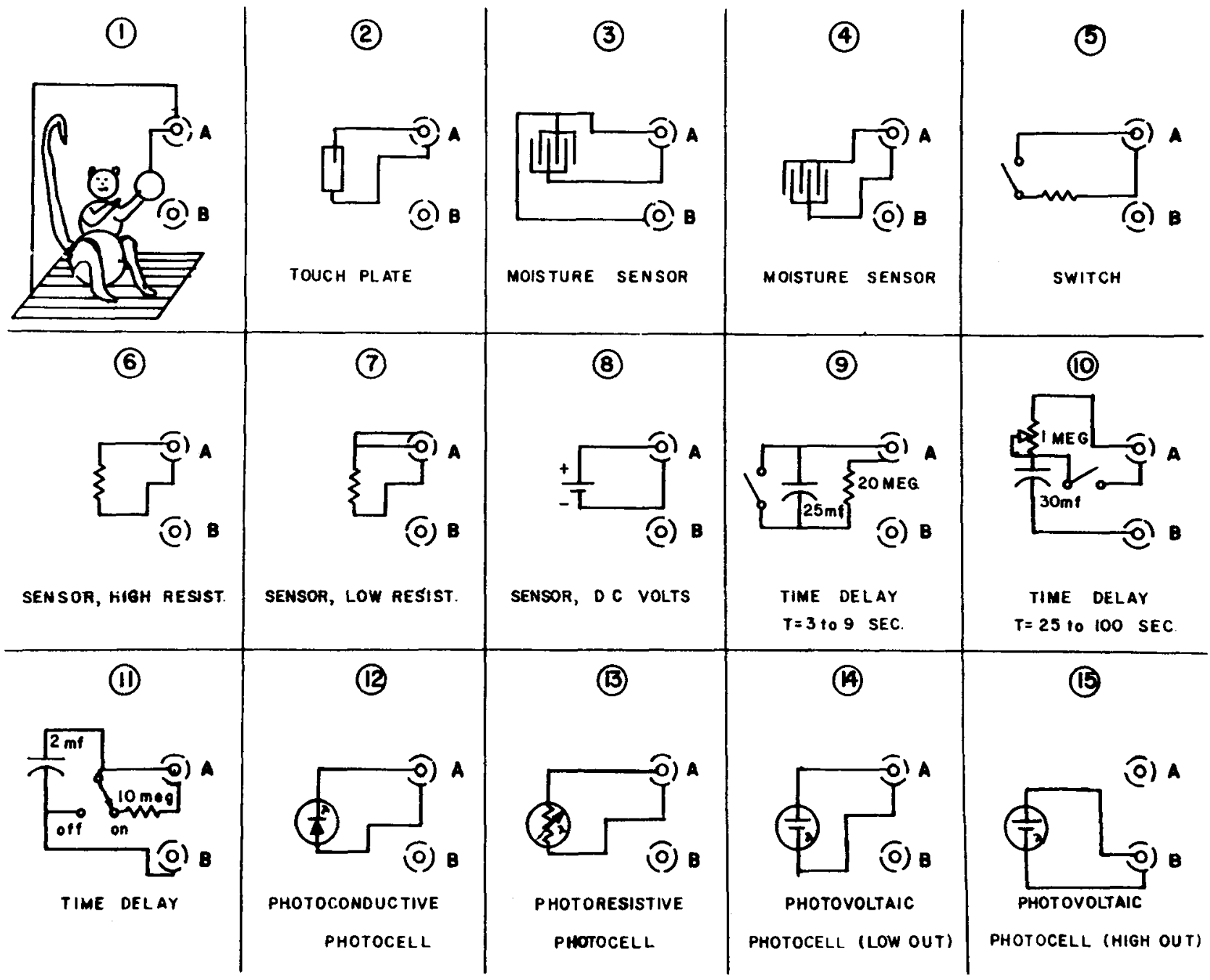

Fig. 4. Various input control devices. 


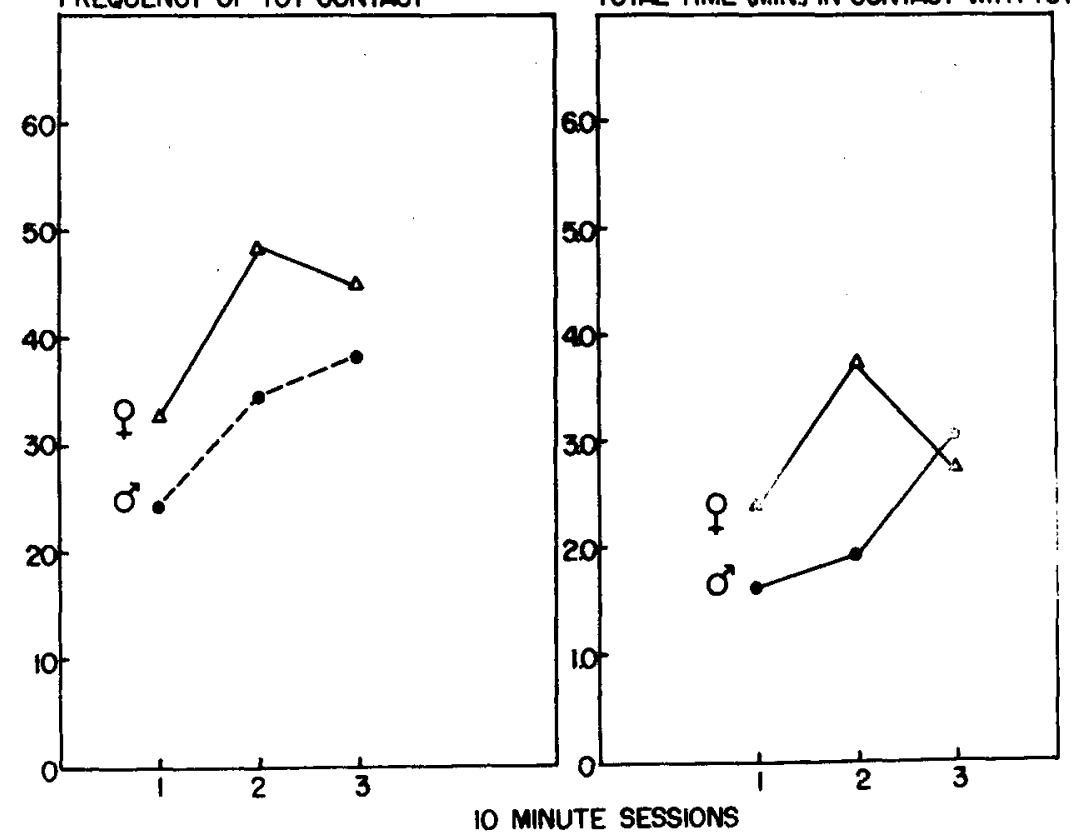

Fig. 5. Toy contact data collected from two isolated infant rhesus monkeys, a male, $\delta$, and a female, $q$, over a $30-\mathrm{min}$ period.

When a signal or bias voltage of proper polarity (base positive with respect to emitter) is applied to the base emitter circuits of $Q 1$ and $Q 2$, the transistors conduct and energize RY-1. The RY-1 is a Sigma dc plate current relay designed for transistor networks with high idle current and applications requiring high release or stable values (cost $\$ 6.50$ each). Bias voltage can be taken from the internal R13, RS circuit or derived externally.

As noted earlier, three basic types of input control devices can be used. Voltage source devices are merely connected to Input $B$, a banana jack receptacle on the front of the box (Fig. 1). Resistor control types are given special consideration. High-resistance devices go to Input $A$ in series with the internal bias circuit; the low-resistance types go to Input B with Input A shorted out. Actually, the resistive devices are made to function like voltage sources since A shorted still has sufficient voltage of proper polarity to forward bias the transistors. The high-resistance range should work on resistances as high as 40 or 50 megohms. The low should operate down to about $1 \mathrm{ohm}$. An approximation of the crossover point is $5 \mathrm{~K}$ ohms.

The switch-type devices can be connected at $A$, and $R 13$ adjusted to provide enough voltage to "pull in" the circuit when the switch is closed. Conversely, the switches can be made to "drop out" the circuit. Shorting $A$ and adjusting R13 to "pull in" the circuit will keep it active until a switch across $B$ causes the network to "drop out" when closed.
The relay can be connected to provide either a normally open or a normally closed control circuit, or both. electrical units on the same line are unfiltered, the lamp on the front of the unit may burn out. If this occurs, the user may need to insert a constant-voltage transformer between the equipment and the ac line or regulate the voltage supply for the Potter and Brumfield genera1-purpose relays, RY-5, RY-6, RY-7, and RY.8.

The output side of the chasis, noted in Fig. 3, contains a separate terminal strip for each channel. These are marked "no" (normally open), "c" (common or relay arm), and "nc" (normally closed). When the relay is not energized the circuit is completed through the $c$ and nc contacts. When energized it is completed through $c$ and no.

Figure 3 shows nine posts making up three isolated contacts per channel. These allow for the recording of up to three separate response measures. Illustrated for simplicity are two time measuring instruments, a Hunter KlocKounter and a Standard Electric clock, and a counter connected to one channel. With slight alteration, banks of timers (Cramer Time Totalizers, Newark Electronics No. 55F444, $\$ 20$ each), and counters (Sedeco, TCe BZ4E, $24 \mathrm{~V}$ dc, $\$ 20$ each) were mounted in a separate chassis and plugged into the output side of the unit to handle all channels.
If the voltage is too high, or large

\section{APPLICATION}

Records taken from two infant rhesus monkeys tested individually are shown in Fig. 5. The male, o, and the female, ?, animals were isolates tested 90 days after birth. A metal toy with attached conductor was placed in each cage to test for responses to a novel object. Frequency of toy contact is indicated on the left and the durations on the right of the figure. The counter and timer were cleared after each 10-min session of the $30 \mathrm{~min}$ noted. The conductor in this case was a pressure-sensitive adhesive tape (Scotch electrical tape, No. X-1170, aluminum foil or copper foil, No. X-1181, 3M Co.).

Plastic toys were tried also; conductivity was achieved by coating the toy with paint (Walsco Copper Print, GC Electronics). Because of possible toxicity effects, however, the painted stimuli were used only for a few minutes at a time. But the number and duration of responses were recorded with little difficulty. Mouth and licking contacts were scored as well as hand manipulations. Of course, plastic is malleable, so it was just a matter of time before the monkeys crinkled or cracked the paint enough to destroy continuity. The technique is described here merely to suggest that conductive paint applications of this type may be useful in other types of research, animal or human.

The apparatus described here is relatively inexpensive if one ignores the cost of the timers and counters. It is also easy to transport (chassis size in inches is 8 high $\times 10$ wide $\times 8$ deep) and versatile enough to use in a variety of experimental situations with different species. A one-channel basic unit less power supply, chassis, connectors, and 3PDT relay, would cost approximately $\$ 12$. (The unit could operate on a battery supply.) A one-channel unit with power supply, 3 PDT relay, chassis, connectors, cabinet, and miscellaneous hardware totals about $\$ 60$. The cost of parts for a complete four-channel unit would be approximately $\$ 130$. Of course, the cost could be cut by building the unit on an open chassis and eliminating such items as connectors, knobs, the cabinet, indicator lights, etc.

\section{NOTES}

1. This work was carried out during the senior author's tenure as Visiting Research Scientist at the Wisconsin Regional Primate Research Center. The cooperation of Professor H.F. Harlow, Director, is gratefully acknowledged.

2. Almost anv photocell will work if connected properly. We have successfully used International Rectifier $53 \mathrm{M}$, $1.1 / 8 \times 1.1 / 8 \times 3 / 16$ in., output $.6 .85 \mathrm{~V}$, $10-16 \mathrm{~mA}(\$ 3.95)$, and Texas Instruments $\mathrm{H}-38$, $0.50 \times 0.82$ in., with an output of 10-100 microamperes (\$4). The TI unit is small, with a very low output, but it performed 
satisfactorily. Both are available from Allied or Newark Electronics, as are photocells manufactured by RCA, Centralab, and Clairex.

3. D3 is a $12-\mathrm{V} 5 \%$ Zener diode made by Texas
Instruments, RCA, International Rectifier, and others. Approximate cost is $\$ 5$.

Any transformer from 24-26 V with center tap is appropriate, e.g., TRIAD F-40X $25.2 \mathrm{~V} \mathrm{ct}$
(\$4.82), TRIAD F-45X $24 \mathrm{~V}$ ct (\$3.77), Chicago-Stancor P-8357 $25.2 \mathrm{~V}$ ct (\$6.55), or KNIGHT $54 \mathrm{~F} 471024 \mathrm{~V}$ ct (\$4.49). All are available from Allied Electronics.
ERRATUM

VALLE, F. P. Sex-typing newborn rats: An improved procedure with Agouti strains. Behavior Research Methods \& Instrumentation, 1970, 2 (4), 205-206.-P. 205, the figures are reversed. What is now Fig. 1 should be Fig. 2, and vice versa. 